\section{P62 IMMUNOLOGICAL ANALYSIS AND CLINICAL PROFILE OF CHRONIC HEPATITIS B VIRUS (HBV) INFECTION IN THE YOUNGER PATIENT}

doi:10.1136/gutjnl-2011-300857a.62

${ }^{1} \mathrm{P}$ T F Kennedy, ${ }^{2} \mathrm{E}$ Sandalova, ${ }^{2} \mathrm{~J}$ Jo, ${ }^{1} \mathrm{U}$ S Gill, ${ }^{1} \mathrm{~L}$ Li, ${ }^{3} \mathrm{I}$ Ushiro-Lumb, ${ }^{4} \mathrm{~S}$ Naik, ${ }^{1} \mathrm{G}$ R Foster, ${ }^{2} \mathrm{~A}$ Bertoletti. ${ }^{1}$ Division of Hepatology, Blizard Institute of Cell \& Molecular Science, Barts and The London School of Medicine and Dentistry, Queen Mary University of London, UK; ${ }^{2}$ Division of Infection and Immunity, Singapore Institute for Clinical Sciences, Singapore; ${ }^{3}$ Department of Virology, Barts and The London NHS Trust, London, UK; ${ }^{4}$ Department of Paediatric Gastroenterology and Hepatology, Barts and The London NHS Trust, London, UK

Introduction Establishing disease phase in chronic HBV is a critical step in long-term disease management. This classification has even more significance at a younger age, where a proportion of eAg positive patients are labelled 'immune tolerant' and treatment is deferred. However, disease phase is diagnosed on clinical phenotype only with little insight into immunological activity.

Aim The aim of this study was to investigate the immunological profile in HBV infected children and young adults across different phases of disease and to compare the HBV-specific responses detected with those of older HBV infected patients.

Method 45 children and young adults (median age 19, range 12-30) were recruited and followed longitudinally for a minimum of 12 months. Clinical profile included ALT, HBV DNA, quantitative HBsAg and, where available, biopsy fibrosis stage and NI scores. This was compared with the immunological analysis for each subject comprising: global T cell cytokine profile (frequency of T cells producing IFN $\alpha$, IL-2, IL-4, TNF $\alpha$ and the ILs; 17, 8, 21, 22 and MIP1a after PMA stimulation), quantity of $\mathrm{T}$ cells (CD4 and CD8) expressing exhaustion markers (PD-1, Lag-3, CTTL-4) and the presence of functional HBV-specific T cells (PBMC stimulated with overlapping HBV peptides covering envelope, core and $\mathrm{E}$ proteins). Results Frequency of $\mathrm{T}$ cells producing immunosuppressive or Th2 cytokines (IL-10, IL-4) and (IL-8, IL-21) is similar between age matched HBV infected and non-infected young adults, while $\mathrm{T}$ cells with pro-inflammatory cytokines (IFN $\alpha$, TNF $\alpha$, IL-17) were increased in the HBV infected group ( $\mathrm{p} \leq 0.05)$. In addition, IL-22 producing cells were present in HBV infected young adults while almost absent in age matched uninfected controls. $T$ cells expressing exhaustion markers were increased in HBV infected patients irrespective of age and correlated with ALT activity. Contrary to predictions HBVspecific T cells were detected with greater frequency in younger HBVinfected subjects when compared with those found in older infected patients $(p \leq 0.05)$. The HBV-specific $\mathrm{T}$ cell response in younger patients show a classical Th1/Tc1 cytokine production profile and expands more vigorously than that seen in older HBV patients.

Conclusion HBV infection in young patients did not induce a generic immune tolerant $\mathrm{T}$ cell cytokine profile. On the contrary, disease activity in chronic HBV appears to be independent of age. Furthermore, the detection of HBV-specific $\mathrm{T}$ cells and the presence of significant liver damage on biopsy in a proportion of our young adult patients underlines the dangers of labelling these patients as 'immune tolerant'. Our findings highlight the dynamic nature of chronic HBV and stress the importance of more formal disease assessment in children and young adults with early therapeutic intervention where indicated.

\section{P63 \\ RIBAVIRIN UPTAKE BY PRIMARY HUMAN HEPATOCYTES CORRESPONDS TO EQUILIBRATIVE NUCLEOSIDE TRANSPORTER 1 EXPRESSION}

doi:10.1136/gutjnl-2011-300857a.63

${ }^{1} \mathrm{~B}$ K Baloch, ${ }^{2} \mathrm{~L}$ Chen, ${ }^{1}{ }^{3} \mathrm{M}$ llyas, ${ }^{2}{ }^{3} \mathrm{~W}$ Irving, ${ }^{2}{ }^{3} \mathrm{~B} \mathrm{~J}$ Thomson. ${ }^{1}$ Department of Pathology, School of Molecular Medical Sciences, University Hospital, Queen's
Medical Centre, University of Nottingham, Nottingham, UK; ${ }^{2}$ Division of Microbiology and Infectious Diseases, School of Molecular Medical Sciences, Oueen's Medical Centre, University of Nottingham, UK; ${ }^{3}$ Nottingham Digestive Diseases Centre Biomedical Research Unit, Nottingham University Hospital Trust NHS, UK

Introduction Combined therapy with pegylated interferon (INF) and ribavirin (RV) is the current standard of care for HCV infection, with a $40 \%$ to $80 \%$ success rate depending on viral genotype. The achievable concentration of RV is an important determinant of therapeutic outcome. A preliminary study used cryopreserved hepatocytes (J Hepatology 2010;52:469-71) to show that equilibrative nucleoside transporter 1 (ENT1) is a major RV transporter. In this study we confirm this observation and demonstrate that the level of ENT1 expression is variable and strongly and directly correlated with RV uptake in primary human hepatocytes. We also provide evidence that ENT1 expression alone may not be sufficient for RV uptake and observe that its levels are not influenced by interferon $\alpha$.

Aim The aim of this study was to assess RV uptake levels and compare it with ENT1 expression in freshly isolated primary hepatocytes. We also aimed to see effects of interferon $\alpha$ on ENT1 expression and ribavirin uptake.

Method Primary hepatocytes were isolated from liver tissue obtained from six human donors. Hepatocytes were cultured on collagen coated plates and exposed to RV diluted in culture medium. Parallel samples were taken for High Performance Liquid Chromatography to assess RV uptake and for quantitative PCR to evaluate ENT1 expression. Similar assays were performed on human hepatoma cell line (Huh7). ENT1 (SLC29A1) gene sequence was analysed by cloning of PCR amplified cDNA followed by direct sequencing. Results We have studied six human livers. As illustrated in Abstract P63 figure 1, there was a strong direct correlation between levels of expression of ENT1 and RV uptake at $24 \mathrm{~h}\left(\mathrm{r}_{\mathrm{s}}=0.94\right.$; $\mathrm{p}$ value $\left.=0.01\right)$ Addition of interferon did not influence RV uptake or ENT1 expression. Strikingly, Huh7 cells expressed ENT1 at similar levels to the majority of primary hepatocytes, but did not take up RV. Sequencing data revealed that ENT1 in Huh7 is wild type.

Conclusion ENT1 has been previously identified as a major transporter involved in RV uptake. In this study, we clearly demonstrate that RV uptake in different sets of primary human hepatocytes is variable and correlates with ENT1 expression. This variation in ENT1 expression may account for differences in response rate in patients receiving anti-HCV therapy. Interferon modulation of ENT1 seems to be a highly unlikely mode of RV and interferon synergistic action in HCV infection. Outcomes in the Huh 7 cell line suggest that, while ENT1 may be necessary, other factors are required to mediate $R V$ uptake.

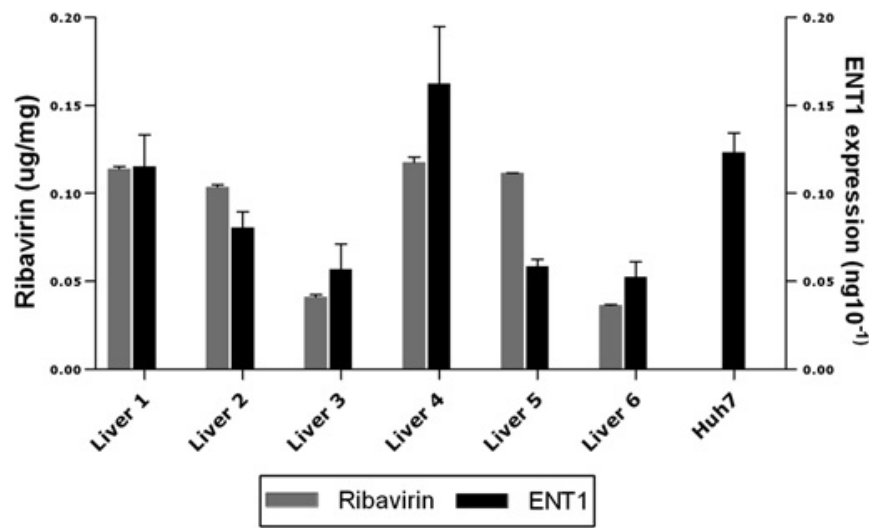

Abstract P63 Figure 1 Correlation of ribavirin uptake and ENT1 expression by primary human hepatocytes and Huh7 cell line. 